\title{
Pathophysiological Basis of Acute Respiratory Failure on Non-Invasive Mechanical Ventilation
}

\author{
C. Romero-Dapueto ${ }^{1}$, H. Budini ${ }^{1}$, F. Cerpa ${ }^{1}$, D. Caceres ${ }^{1}$, V. Hidalgo ${ }^{1}$, \\ T. Gutiérrez ${ }^{1}$, J. Keymer ${ }^{1}$, R. Pérez ${ }^{1}$, J. Molina ${ }^{2}$ and C. Giugliano-Jaramillo*,1 \\ ${ }^{I}$ Servicio de Medicina Física y Rehabilitación, Clínica Alemana de Santiago, Santiago, Chile \\ ${ }^{2}$ Escuela de Kinesiología, Universidad del Desarrollo, Santiago, Chile
}

\begin{abstract}
Noninvasive mechanical ventilation (NIMV) was created for patients who needed noninvasive ventilator support, this procedure decreases the complications associated with the use of endotracheal intubation (ETT). The application of NIMV has acquired major relevance in the last few years in the management of acute respiratory failure (ARF), in patients with hypoxemic and hypercapnic failure. The main advantage of NIMV as compared to invasive mechanical ventilation (IMV) is that it can be used earlier outside intensive care units (ICUs). The evidence strongly supports its use in patients with COPD exacerbation, support in weaning process in chronic obstructive pulmonary disease (COPD) patients, patients with acute cardiogenic pulmonary edema (ACPE), and Immunosuppressed patients. On the other hand, there is poor evidence that supports the use of NIMV in other pathologies such as pneumonia, acute respiratory distress syndrome (ARDS), and during procedures as bronchoscopy, where its use is still controversial because the results of these studies are inconclusive against the decrease in the rate of intubation or mortality.
\end{abstract}

Keywords: Acute pulmonary edema, acute respiratory failure, COPD, immunosuppress patients, non-invasive ventilation.

\section{INTRODUCTION}

The Non Invasive Mechanical Ventilation (NIMV) is any form of administration of positive pressure using an interface or a face mask, without the need of using an endotracheal tube (ETT) [1]. Since the early twentieth century, the need for a less invasive ventilation support for patients is created, thus, reducing the complications associated with the use of ETT, being Meduri et al. the first to describe the use of mask ventilation to avoid endotracheal intubation. They compared 10 patients, 6 of them with diagnosis of COPD, 2 with congestive heart failure (CHF) and 2 with pneumonia avoiding the need for intubation in 8 cases [2].

The application of NIMV has become more important in recent years in the management of acute respiratory failure (ARF), both in patients with hypoxemic and hypercapnic respiratory failure. The great advantage over invasive mechanical ventilation (IMV) is that it can be used early outside the ICU by trained and successful short-term staff [3].

The Acute Respiratory Failure (ARF) is a syndrome characterized by the inability of the respiratory system to maintain adequate arterial $\mathrm{O}_{2}$ and $\mathrm{CO}_{2}$ levels according to the demands of cellular metabolism. The ARF may be caused by the failure of the exchanger, lung, organ or pump, or failure of the respiratory muscles. The ARF can be classified by the dysfunctional element or the evolution time when the condition occurs.

*Address correspondence to this author at the Servicio de Medicina Física y Rehabilitación, Clínica Alemana de Santiago, Avenida Vitacura 5951, Vitacura, Santiago, Chile; Tel: +56222101421; Fax: +56222101421 ;

E-mail: cgiuglianoj@alemana.cl
Hypoxemic ARF is the most common form of acute respiratory failure. Hypoxemic ARF occurs by an alteration in the ventilation/perfusion ratio, often resulting from the presence of alveolar infiltrates and/or lung collapse. It is characterized by low blood pressure of oxygen $\left(\mathrm{PO}_{2}\right)$, normal or low blood pressure of carbon dioxide $\left(\mathrm{PCO}_{2}\right)$. Hypercapnic ARF occurs when there is a decrease in ventilation drive, neuromuscular disorders or increased work of breathing, usually due to bronchial obstruction. It is characterized by high $\mathrm{PCO}_{2}(>45 \mathrm{~mm} \mathrm{Hg})$ with normal or low $\mathrm{PO}_{2}\left(\mathrm{PO}_{2}<\right.$ or $\left.=60\right)$. Respiratory failure sometimes is deemed chronic when it occurs in patients during several days or longer, or can be acute-on -chronic when the gas exchange suffers deterioration from this basal status [4].

This classification is not static, and sometimes clinicians found mixed disorders because the lungs and heart do not work separately $[4,5]$. See Fig. (1).

\section{HYPERCAPNIC RESPIRATORY FAILURE}

The main NIMV objective in hypercapnic ARF is to increase the alveolar ventilation and decrease the hypoventilation, resulting in high $\mathrm{PaCO}_{2}$, thus, relieving the burden of respiratory muscles with the consequent reduction/normalization of arterial $\mathrm{pH}$ while the trigger for respiratory failure problem is solved.

\subsection{COPD and NIMV}

Chronic Obstructive Pulmonary Disease (COPD) is one of the diseases where there is more evidence against the use of NIMV to treat exacerbations. The pathophysiological 


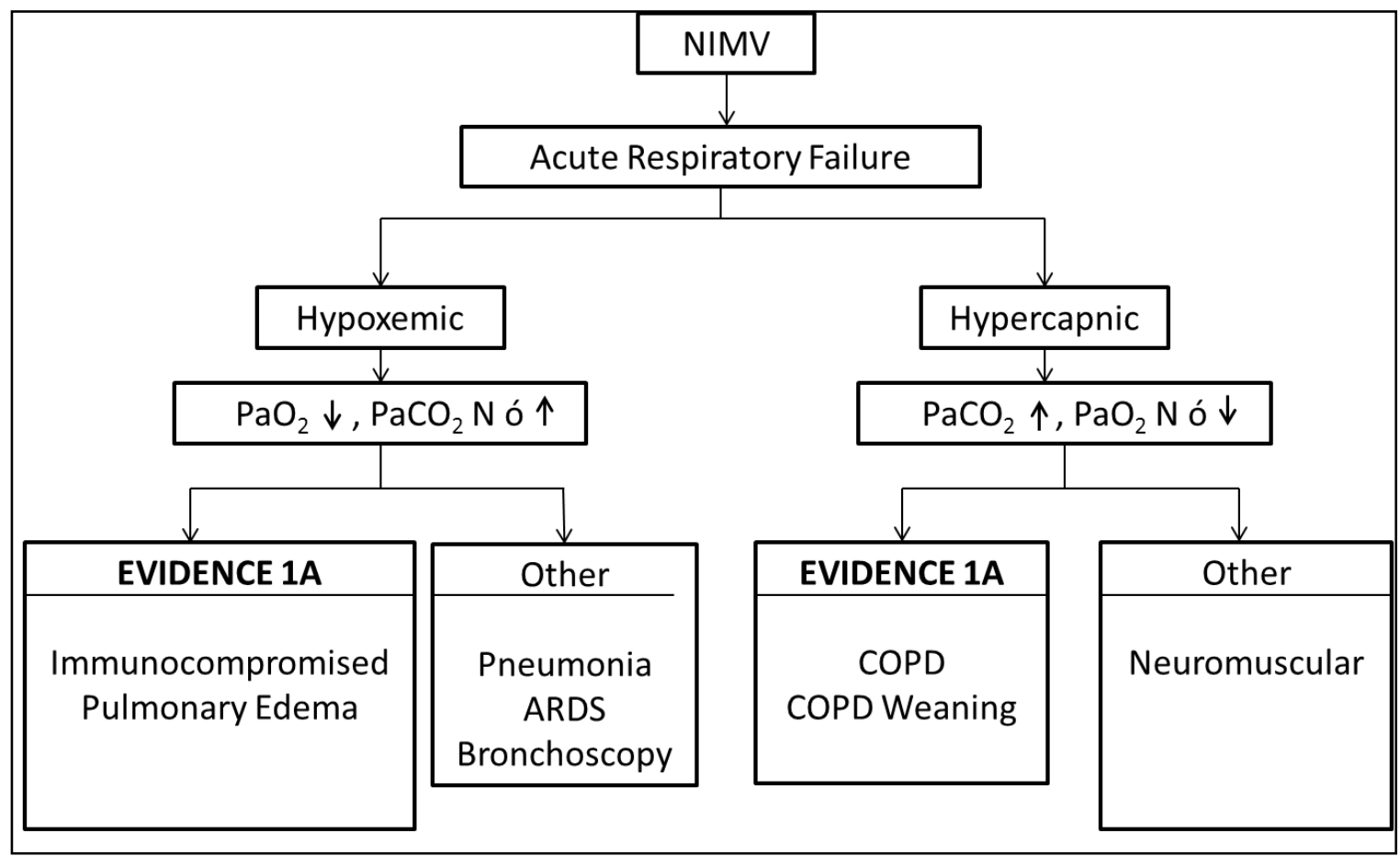

Fig. (1). Acute Respiratory Failure classification. $\mathrm{NIMV}=$ Non invasive mechanical ventilation, $\mathrm{PaO}_{2}=\mathrm{Oxygen}_{\text {arterial }}$ pressure, $\mathrm{PaCO} \mathrm{C}_{2}=$ Carbon dioxide arterial pressure, ARDS $=$ Acute respiratory distress syndrome, $\mathrm{COPD}=$ Chronic obstructive pulmonary disease.

basis for the use of NIMV in COPD exacerbations is to reduce hypoventilation and improve hypoxemia. The most accepted hypothesis is by reducing the work of breathing, facilitating recovery from fatigue of the respiratory muscles [6].

Other studies postulate that hypercapnia is reduced by the combination of decreasing the load of the respiratory muscles, decreasing lung deflation assisted by the NIMV, the displacement of more proximal isopressure point and to the uncollapsed airways, and decreased respiratory input associated with the central respiratory mechanism. Summarizing the more suitably explanation for the pathophysiological utility of NIMV in COPD is the reduction of dynamic hyperinflation using positive pressure [7]. Other studies suggest a decrease in airway resistance due to the application of positive intrathoracic pressure, thus reducing edema of the airway wall. Changes in the resistance of the wall in the airway may be given by the recruitment of previously collapsed alveolar units by the hyperinflation of the normal emphysematous areas [7].

According to the review of Keenan, in 2009 [5] NIMV offers greater absolute reduction in the ARF versus reduction associated with the use of IMV in COPD patients also reduces intubation and mortality in patients with severe disease who have exacerbations. The use of NIMV in COPD exacerbation is also recommended (evidence type A) [5]. It also describes which should be the first line therapy in COPD patients with acute respiratory distress syndrome and respiratory acidosis. It also proposes how to determine the best modality or patient interface, controversial topic, because different publications show dissimilar benefits associated with different ventilation modes.
Several randomized controlled trials have also compared the use of NIMV by using standard oxygen therapy, showing comparative advantages in the use of NIMV in relation to an increase in gas exchange and associated clinical symptoms $[8,9]$. However, other studies show that early use of NIMV, before respiratory acidosis appears, improve treatment outcomes [6].

\subsection{Weaning and Respiratory Failure After Extubation in COPD Patients and NIMV}

The main use of NIMV in weaning is to prevent extubation failure in COPD patients who also have hypercapnia or heart failure. Several randomized studies show that, the weaning of IMV in stable COPD patients with hypercapnic failure is a safe technique, and also shows effectiveness in those who failed on spontaneous ventilation trial [10].

In most cases, when NIMV is applied immediately after extubation in patients at high risk (elderly patients with heart failure or failed before the spontaneous breathing test, etc) significantly decreases the rate of reintubation [11]. However, other studies have shown that there is no decrease in the rate of intubation, but on the contrary, delaying the intubation of patients when needed and have been given the chance NIMV, increases mortality in this group patients and days of stay in ICU. Successful treatment of respiratory failure after extubation will depend on the etiology of the patient, thus showing better results in COPD and acute cardiogenic pulmonary edema (ACPE), because of the pathophysiological mechanisms mentioned above [11-13].

From the pathophysiological point of this view, what NIMV achieved in this group of patients is an increase in 
tidal volume (VT), diminish respiratory rate, and an improvement in gas exchange. So, Hill in 2008, describes in his review that the ideal patients for NIMV post extubation are patients with COPD, as the NIMV counteracts the fatigue of the respiratory muscles due to underlying diagnosis, tachypnea and increased work of breathing, therefore, corrects the undesired increase of autoPEEP [14].

\section{HYPOXEMIC RESPIRATORY FAILURE}

The application of positive pressure in hypoxemic ARF produces decreased intrapulmonary shunt and thereby improves oxygenation. This occurs by the recruitment of collapsed alveoli, prevents collapse previously opened by the positive pressure, increase FRC and improve balance of the $\mathrm{V} / \mathrm{Q}$.

\subsection{Immunosuppressed Patients and NIMV}

The acute respiratory failure is the main cause of admission of hematological patients to ICU, where transplanted patients with immunosuppressive therapy or malignant tumors, had a mortality rate of $40 \%$ and $60 \%$ respectively [15]. In these groups, the immunosuppressed patients usually develop hypoxemic respiratory failure [16]. These types of patients include; neutropenia after chemotherapy, or bone marrow transplantation, patients with hematologic cancers, drug-induced immunosuppression during organ transplants or corticosteroid therapy, or by acquired immunodeficiency syndrome [16]. In this patient population, the NIMV objective is to ensure adequate $\mathrm{PaO}_{2}$, while the cause of acute decompensation arrives. The reason for the decrease in oxygenation is the collapse in the distal alveolar spaces and/or its occupation. This results in a decrease in ventilation/perfusion ratio, which can sometimes behave like shunt, leading to hypoxemia. If this group of patients develops sepsis with non-pulmonary origin, it can induce an acute respiratory failure by capillary action, and as result increases oxygen requirement and increase $\mathrm{CO}_{2}$ concentration. If the acute respiratory failure is a primary pulmonary disease, it is caused by direct pulmonary infiltration [17].

Respiratory failure by systemic inflammatory response syndrome (SIRS) in hematologic patients is caused by capillary leakage due to alterations in the microcirculation, endothelial dysfunction, and oxidative stress caused by cytokines, complement system and coagulation disorders [17].

Pulmonary infiltrates are caused by the migration of inflammatory cells to lung tissue. However, in severe leucopenia, pulmonary infiltrates are developed without visible pulmonary infiltrates [17].

During treatment with NIMV, there are no differences in the development of treated respiratory failure and mortality depending on its cause, primary or secondary to SIRS [17]. NIMV improves oxygenation, and thus decreases the rate of intubation and mortality, mainly in immunosuppressed patients with ARF or pneumonia when compared with single use of oxygen $[16,18]$. Also in patients with neutropenia respiratory events occur in half of the cases, half of which develop ARF, where the NIMV reduces complications of endotracheal intubation and IMV $[17,19]$.

The positive end expiratory pressure (PEEP) redistributes the extravascular fluid, improves alveolar recruitment, and decreases atelectasis. The pressure support (PS) decreases the work of breathing, and helps maintain an adequate tidal volume with correct alveolar ventilation, and it allows recovering the efficiency of the respiratory muscles [16].

In patients with acquired immunodeficiency syndrome the most common cause of admission to ICU is acute respiratory failure, caused by Pneumocystis jirovesi. In these patients falling IMV for more than 5 days develops nosocomial infections and pneumothorax, which are mortality predictors at 3 months. The NIMV, especially CPAP improves gas exchange, respiratory rate and dyspnea, and this decreases the rate of endotracheal intubation by $67 \%$ and thus mortality [20].

Therefore, the early use of NIMV in patients with hematologic disorders, shares changes in ventilation parameters, preventing the progression to acute lung injury, thus also avoiding the need for mechanical ventilation and admission to the ICU, and therefore, decreasing associated complications and mortality $[15,18]$.

\subsection{Acute Cardiogenic Pulmonary Edema and NIMV}

Acute pulmonary cardiogenic edema (ACPE) is the result of heart failure, which results in an increase in retrograde pressure in the pulmonary circulation, which triggers an increase in the extravasation of fluid into the alveoli, thus being unable to be completely removed by the lymphatic system, and thereby generating an increase in intrapulmonary hydrostatic pressure liquid, thus, generating edema. Moreover, edema acts on pulmonary surfactant inactivating and diluting due to the increase of liquid, in this manner altering its properties to prevent lung collapse, decreasing pulmonary distensibility, making it less distensible. Thus, pathophysiology, loss of the elastic properties of lung causes intrapulmonary shunt, and favoring the appearance of alterations in ventilation-perfusion $(\mathrm{V} / \mathrm{Q})$ ratio.

The APCE is one of the most common and best evidences in the literature for the causes of acute hypoxemic respiratory failure. In insignificant number of cases, the standard of care is insufficient to address the aforementioned disorders, and need to assist patients with ventilation therapy, which seeks to reverse the acidosis, increased ventilation work, hypoxemia and/or hypercapnia that patients may present.

There are various techniques for administering positive pressure, such as CPAP Bousignac [21], NIMV in CPAP mode, which presents better efficiency for easy use and bilevel, which has proven to consistently improve respiratory muscle deficiency [22].

The main mechanism to improve respiratory and cardiac function in addition to oxygenation is by alveolar recruitment and increased functional residual capacity (FRC), thus decreasing the intrapulmonary shunt. The increase in intrathoracic pressure determined by the use of NIMV in bi-level mode is by ventilation assistance during 
inspiration, thus reducing the burden of respiratory muscles during the acute phase of APCE and thus preventing muscle fatigue, with and subsequent drop in blood gases and $\mathrm{PaO}_{2}$. To improve gas exchange, it begins to reverse acidosis, resulting in a direct effect on cardiac output, and decrease in back pressure and the passage of fluids into the alveolar space edema production [23].

The review of Cochrane 2013 says that the main effect of NIMV is to prevent alveolar collapse, redistributing intraalveolar fluid, thus improving pulmonary compliance and reducing the pressure deflection to improve ventilation work. Therefore, to prevent alveolar collapse by using positive end-expiratory pressure, also reduces preload and afterload, thereby improving ventricular function.

\section{OTHER USES OF NIMV}

There are other uses within NIMV, but these have no evidence level A, and are not well studied yet.

\subsection{Pneumonia and NIMV}

Pneumonia is an infection caused by an endogenous and/or exogenous pathogen; with a consequent acute lung inflammation characterized by the presence of alveolar infiltrates. Due to alveolar occupation in pneumonia, there are high levels of intrapulmonary shunt and a decrease in the FRC.

The pathophysiological effect of the NIMV is by decrease pneumonia, shunt and thereby improving oxygenation. This occurs by the opening and expansion of collapsed alveoli, besides preventing collapse previously opened by the positive pressure, it increases FRC and improves balance of the $\mathrm{V} / \mathrm{Q}$.

Few studies have mentioned the use of NIMV on community-acquired pneumonia; its use is controversial due to the high variability in the rates of failure. Of all failure predictors the most commonly described is the non-variation or decrease in $\mathrm{PaO}_{2}$ with the use of NIMV [18, 24].

Some authors report that by delaying the endotracheal intubation, there may occur an increase in mortality when applying NIMV in patients with community-acquired pneumonia [25].

Studies also differ on the evolution of NIMV in terms of the presence of comorbidities such as respiratory or cardiac failure [18]. In these cases, they described less NIMV failure to those without these underlying conditions [18].

In the same way, there are studies in patients with severe community acquired pneumonia, with hypoxemic respiratory failure that when are subjected to NIMV, has a lower rate of endotracheal intubation and ICU mortality [5].

The recommendations from the few available studies on the use of NIMV in pneumonia suggest that; in patients with ARF associated with community-acquired pneumonia, and with predictors of failure of NIMV, intubation and conventional mechanical ventilation should not be postponed to decrease mortality [18].

\subsection{Acute Respiratory Distress Syndrome and NIMV}

The acute respiratory distress syndrome (ARDS) is defined as an "acute, diffuse inflammatory lung injury, which results in an increased pulmonary vascular permeability, increased lung weight, and loss of aerated lung tissue. Clinical markers of ARDS are hypoxemia and bilateral radiographic opacities associated with increased mixed venous blood, increased physiological dead space, and decreased lung compliance" [26]. ARDS is characterized by tachypnea, severe dyspnea, cyanosis, refractary to oxygen hypoxemia, decreased lung compliance, and alveolar infiltrates on chest radiograph [26]. Within the pathophysiological mechanisms associated with ARDS, two phenomena occur; a diffuse lung inflammatory process and acute pulmonary edema due to an increased alveolarcapillary permeability.

In acute phase, inflammation predominates, with infiltration of polymorphonuclear leukocytes and cell destruction. There is interstitial and alveolar edema due to increased capillary permeability, but equally there are atelectasis associated with edematous weight gain lung [27].

From the viewpoint of respiratory mechanics, acute phase is characterized by a restrictive condition, with decreased FRC, due to alveolar collapse and replacement from air to liquid and also decreases lung compliance [27].

Few studies support the use of NIMV in ARDS, finding decrease in respiratory rate and increase in $\mathrm{PaO}_{2} / \mathrm{FiO}_{2}$, preventing endotracheal intubation, reducing the incidence of septic shock and increasing survival, but this is mainly in the early stage of ARDS, in patients with no or minimal organ dysfunction $[18,28]$, because the application of positive pressure is able to reduce intrapulmonary shunt, and thereby improve oxygenation. The positive inspiratory pressure can recruit alveolar units, positive end expiratory pressure (PEEP) maintains this recruitment, increasing FRC and improving the balance between ventilation and perfusion.

There are several studies that evaluate the use of NIMV in ARDS, but these are not strong in supporting the systematic use of NIMV, finding no decrease in the rate of endotracheal intubation or mortality [5, 18, 29].

The election treatment of ARDS is intubation and mechanical ventilation, NIMV is controversial because according to several authors ARDS is a predictor of failure of NIMV [28].

The Berlin Consensus proposed besides the new definition, treatment strategies depending on the severity of ARDS, suggesting for mild ARDS the use of NIMV [26]. Studies also suggest that if NIMV is used either in a select group of patients, since shock, metabolic acidosis, and severe hypoxemia are predictors of NIMV failure [18]. All this under close monitoring due to the high risk of failure, without delaying the introduction of invasive mechanical ventilation in case of failure [29].

\subsection{Fibrobronchoscopy Procedure and NIMV}

During bronchoscopy, the diameter of the bronchoscope plays an important role in airway obstruction. In non- 
Table 1. Clinical and mechanistic evidence that support the use of NIMV.

\begin{tabular}{|c|c|c|}
\hline & NIMV Evidence & NIMV Pathophysiology \\
\hline \multirow{4}{*}{ COPD } & \multirow{4}{*}{ A } & Decreases dynamic Hyperinflation \\
\hline & & Reduces Hypoventilation and improves hypoxemia \\
\hline & & Decreases the load of the respiratory muscles \\
\hline & & Improves the work of breathing \\
\hline \multirow{4}{*}{ COPD Weaning } & \multirow{4}{*}{ A } & Decrease the rate of intubation \\
\hline & & Increases tidal volumen $(\mathrm{Vt})$ \\
\hline & & Decreases respiratory rate \\
\hline & & Improves gas exchange \\
\hline \multirow{3}{*}{ Cardiogenic Pulmonary Edema } & \multirow{3}{*}{ A } & Reverses the acidosis \\
\hline & & Increases ventilatory work \\
\hline & & Improves hypoxemia and decreases hypercapnia \\
\hline \multirow{3}{*}{ Immunocompromised } & \multirow{3}{*}{ A } & Decreases intrapulmonary shunt \\
\hline & & Increases Functional residual capacity (FRC) \\
\hline & & Ensure adequate $\mathrm{PaO}_{2}$, while the cause of acute decompensation is solved \\
\hline \multirow{5}{*}{ Bronchoscopy } & \multirow{5}{*}{ B } & Decreases intrapulmonary shunt \\
\hline & & Improves oxygenation \\
\hline & & Increases functional residual capacity (FRC) \\
\hline & & Decreases the incidence of complications \\
\hline & & Avoid ARF after Bronchoscopy \\
\hline \multirow{3}{*}{ Pneumonia } & \multirow{3}{*}{$\mathrm{C}$} & Decreases intrapulmonary shunt \\
\hline & & Improves oxygenation \\
\hline & & Increases Functional residual capacity (FRC) \\
\hline \multirow{3}{*}{ ARDS } & \multirow{3}{*}{$\mathrm{C}$} & Decreases intrapulmonary shunt \\
\hline & & Improves oxygenation \\
\hline & & Increases functional residual capacity (FRC) \\
\hline
\end{tabular}

intubated patients the bronchoscope occupies approximately $10 \%$ of the internal diameter of the trachea, thus decreasing the tidal volume and increased work of breathing [30]. This is associated with impaired respiratory mechanics and gas exchange causing hypoxemia and hypercapnia.

Applying suction during the bronchoscopy, the endexpiratory volume, and the positive end expiratory pressure (PEEP) are diminished, thereby facilitating alveolar closing and the venous admission, harmful for lung mechanics. After performing the bronchoscopy, time normalization can take several hours in patients with severe parenchymal lung disorders such as fibrosis, or inflammation of the parenchyma that alter gas exchange and produces hypoxemia [31].

Blood pressure of oxygen normally decreases between 10-20 $\mathrm{mmHg}$ in patients undergoing uncomplicated bronchoscopy, in hypoxemic patients there is great risk of developing respiratory failure or cardiac arrhythmias.

NIMV works in the treatment of acute respiratory failure, improving gas exchange abnormalities and reducing the signs of labored respiration, dyspnea, and activity of accessory respiratory muscles [32]. During the performance of bronchoscopy, the use of NIMV prevents the development of hypoxemia, and so acute respiratory failure, especially in patients with exacerbations of chronic respiratory disease like COPD, acute cardiogenic pulmonary edema and immunocompromised patients [33, 34] in this context, NIMV should be the first choice of ventilation support for these patients that have evidence $1 \mathrm{~A}$. NIMV reduces rates of intubation, length of hospital stay, morbidity and mortality in those patients [31].

In patients with severe hypoxemia $\left(\mathrm{PaO}_{2} / \mathrm{FiO}_{2}\right.$ below 200) NIMV improves gas exchange during and after the bronchoscopy, thus decreasing the incidence of complications [31].

Based on the foregoing, it can be said that the bronchoscopy is a procedure used for diagnostic and therapeutic purposes in critical patients units. The use of NIMV in patients who undergo bronchoscopy has supporting evidence behind it and its use is an alternative in severe patients, and prevents complications related to the 
endotracheal intubation and invasive mechanical ventilation [35].

In Baumann et al. study published in Critical Care 2011, found that only $10 \%$ of patients undergoing bronchoscopy with NIMV required endotracheal intubation at 8 hours performed the procedure because of complications related to the technique of bronchoscopy [35].

\section{CONCLUDING REMARKS}

The use of NIMV helps recover hypercapnic ARF in COPD patients, mainly because counteracting the dynamic hyperinflation and the autoPEEP that these patients generate. The result promotes the rest of the respiratory muscles, thus, reducing the work of breathing and dyspnea and also produces a gasometric improvement due to correction of $\mathrm{PaCO}_{2}$.

By contrast in hypoxemic patients, the evidence supports the use of NIMV only in immunosuppressed patients and ACPE, which should be the first line of treatment for correction of $\mathrm{PaO}_{2}$. In immunosuppressed patients, the use of NIMV reduces intrapulmonary shunt, improving oxygenation, and thereby avoiding the ETT and its associated complications. In ACPE instead $\mathrm{PaO}_{2}$ improved by decreasing the pre- and afterload, increasing venous return and thus improve cardiac function. With the foregoing, the backpressure decreases lung edema by decreasing production and as in immunosuppressed patients, shunts also decreases.

There is little evidence that support the use of NIMV in other diseases such as pneumonia and ARDS, in which the studies are not conclusive to the hard endpoint as decreasing the rate of intubation and mortality, as most agree that often delays the ETT and this increases the complications and ultimately mortality. Use with strict surveillance and monitoring is suggested, so that if failure criteria are presented notify early and do not delay intubation.

The knowledge and use of NIMV would be undervalued relative to its pathophysiology in the clinical setting, as there is strong evidence that endorses and many studies do not use it as the first-line of treatment (Table 1). Moreover from the health perspective, it lowers health care costs associated with a decreased ICU days, days of IMV, patient sedation and total hospital stay.

\section{CONFLICT OF INTEREST}

The authors declare that there are no conflict of interest.

\section{ACKNOWLEDGEMENTS}

Author's Role: All authors provided support in writing, proofreading and approving the final versión of this manuscript. A special thanks to Jeronimo Graf and ICU of Clínica Alemana de Santiago.

\section{REFERENCES}

[1] Hess D. Noninvasive ventilation for acute respiratory failure. Respir Care 2013; 58: 950-72.
[2] Pierson D. History and epidemiology of noninvasive ventilation in the acute-care setting. Respir Care 2009; 54: 40-52.

[3] Goodacre S, Stevens J, Pandor A, et al. Prehospital noninvasive ventilation for acute respiratory failure: systematic review, network meta-analysis, and individual patient data meta-analysis. Acad Emerg Med 2014; 21: 960-70.

[4] Williams J, Cox C, Hargett C, et al. Noninvasive positive-pressure ventilation (NPPV) for acute respiratory failure. Rockville (MD): Agency for Healthcare Research and Quality (US); 2012 Jul. Report No.: 12-EHC089-EF. AHRQ Comparative Effectiveness Reviews.

[5] Keenan S, Mehta S. Noninvasive ventilation for patients presenting with acute respiratory failure: the randomized controlled trials. Resp Care 2009; 54: 116-26.

[6] Mas A, Masip J. Noninvasive ventilation in acute respiratory failure. Int J Chron Obstruct Pulmon Dis 2014; 11: 837-52.

[7] Díaz O, Bégin P, Torrealba B, Jover E, Lisboa C. Effects of noninvasive ventilation on lung hyperinflation in stable hypercapnic COPD. Eur Respir J 2002; 20: 1490-8.

[8] Brochard L, Mancebo J, Wysocki M, et al. Noninvasive ventilation for acute exacerbations of chronic obstructive pulmonary disease. N Engl J Med 1995; 333: 817-22.

[9] Celikel T, Sungur M, Ceyhan B, Karakurt S. Comparison of noninvasive positive pressure ventilation with standard medical therapy in hypercapnic acute respiratory failure. Chest 1998; 114: $1636-42$.

[10] Ferrer M, Valencia M, Nicolas JM, et al. Early non-invasive ventilation averts extubation failure in patients at risk: a randomized trial. Am J Respir Crit Care Med 2006; 173: 164-70.

[11] Nava S, Hill N. Non-invasive ventilation in acute respiratory failure. Lancet 2009; 374: 250-9.

[12] Elliott M, Confalonieri M, Nava S. Where to perform noninvasive ventilation? Eur Respir J 2002; 19: 1159-66.

[13] Barbè F, Togores B, Rubi M, et al. Noninvasive ventilatory support does not facilitate recovery from acute respiratory failure in chronic obstructive pulmonary disease. Eur Respir J 1996; 9: 1240-5.

[14] Burns K, Meade M, Premji A, Adhikari N. Noninvasive positivepressure ventilation as a weaning strategy for intubated adults with respiratory failure. Cochrane Database Syst Rev 2013; 9(12): CD004127.

[15] Lemiale V, Resche-Rigon M, Azoulay E. Early Non-invasive ventilation for acute respiratory failure in immunocompromised patients (ivnictus): study protocol for a multicenter randomized controlled trial. Trials 2014; $15: 372$.

[16] Hilbert G, Gruson D, Vargas F, et al. Noninvasive ventilation in immunosuppressed patients with pulmonary infiltrates, fever, and acute respiratory failure. N Engl J Med 2001; 344: 481-7.

[17] Razlaf P, Pabst D, Mohr M, et al. Non-invasive ventilation in immunosuppressed patients with pneumonia and extrapulmonary sepsis. Respir Med 2012; 106: 1509-16.

[18] Hess DE, Fessler HE. Respiratory controversies in the critical care setting. should noninvasive positive-pressure ventilation be used in all forms of acute respiratory failure? Respir Care 2007; 52: 56878.

[19] Azoulay E, Lemiale V. Non-invasive mechanical ventilation in hematology patients with hypoxemic acute respiratory failure: a false belief? Bone Marrow Transpl 2012; 47: 469-72.

[20] Confalonieri M, Calderini E, Terraciano S, et al. Noninvasive ventilation for treating acute respiratory failure in aids patients with pneumocystis carinii pneumonia. Intensive Care Med 2002; 28: 1233-8.

[21] Sehlin M, Törnell S, Öhberg F, Johansson G, Winsö O. Pneumatic performance of the Boussignac CPAP system in healthy humans. Resp Care 2011; 56: 818-26.

[22] Vital F, Ladeira M, Atallah A. Non-invasive positive pressure ventilation (CPAP or bilevel NPPV) for cardiogenic pulmonary edema. Cochrane Database Syst Rev 2013; 31: CD005351.

[23] Minuto A, Giacomini M, Giamundo B, et al. Non-invasive mechanical ventilation in patients with acute cardiogenic pulmonary edema. Minerva Anestesiol 2003; 69: 835-40.

[24] Carrillo A, Gonzalez-Diaz G, Ferrer M. Non-invasive ventilation in community-acquired pneumonia and severe acute respiratory failure. Intensive Care Med 2012; 38: 458-66.

[25] Mas A, Masip J. Noninvasive ventilation in acute respiratory failure. Int J COPD 2014; 9: 837-52. 
[26] Arancibia F. Nueva definición de Berlín de Síndrome de Distrés Respiratorio Agudo. Revista chilena de medicina intensiva 2012; 27: $35-40$

[27] Matthay M, Ware L, Zimmerman G. The acute respiratory distress syndrome. J Clin Invest 2012; 122: 2731-40.

[28] Agarwal R, Aggarwal A, Gupta D. Lung injury/acute respiratory distress syndrome: a proportion meta-analysis. Resp Care 2010; 55: 1653-60.

[29] Berg K, Clardy P, Donnino M. Noninvasive ventilation for acute respiratory failure: a review of the literature and current guidelines. Int Emerg Med 2012; 7: 539-45.

[30] Estella A. Bronchoscopy in Mechanically Ventilated Patients. Global Perspectives on Bronchoscopy. INTECH Open Access Publisher 2012.
[31] Antonelli M. Noninvasive positive-pressure ventilation vs conventional oxygen supplementation in hypoxemic patients undergoing diagnostic bronchoscopy. Chest 2002; 121: 1149-54.

[32] Esquinas A, Zuil M, Scalac R, Chinerd E. Broncoscopia durante la ventilación mecánica no invasiva: revisión de técnicas y procedimientos. Arch Bronconeumol 2013; 49: 112-5.

[33] Peñuelas O, Frutos-Vivar F, Esteban A. Noninvasive positivepressure ventilation in acute respiratory failure. CMAJ 2007; 177: 1211-8.

[34] Hill N, Brennan J, Garpestad E, Nava S. Noninvasive ventilation in acute respiratory failure. Crit Care Med 2007; 35: 2402-7.

[35] Baumann H, Klose H, Simon M, et al. Fiber optic bronchoscopy in patients with acute hypoxemic respiratory failure requiring noninvasive ventilation - a feasibility study. Crit Care 2011; 15 : R179. 